\title{
A Self-Stabilizing 3-Approximation for the Maximum Leaf Spanning Tree Problem in Arbitrary Networks
}

\author{
Sayaka Kamei ${ }^{1 \star}$, Hirotsugu Kakugawa ${ }^{2 \star \star}$, Stéphane Devismes ${ }^{3}$, and Sébastien \\ Tixeuil $^{4 \star \star \star}$ \\ 1 Dept. of Information Engineering, Hiroshima University, Japan, \\ E-mail: s-kamei@se.hiroshima-u.ac.jp \\ 2 Dept. of Computer Science, Osaka University, Japan, \\ E-mail: kakugawa@ist.osaka-u.ac.jp \\ 3 Université Joseph Fourier, Grenoble I, France, E-mail: Stephane. Devismes @imag. fr \\ 4 LIP6 UMR 7606, Université Pierre et Marie Curie, France, \\ E-mail: Sebastien.Tixeuil@lip6.fr
}

\begin{abstract}
The maximum leaf spanning tree (MLST) is a good candidate for constructing a virtual backbone in self-organized multihop wireless networks, but is practically intractable (NP-complete). Self-stabilization is a general technique that permits to recover from catastrophic transient failures in self-organized networks without human intervention. We propose a fully distributed self-stabilizing approximation algorithm for the MLST problem on arbitrary topology networks. Our algorithm is the first self-stabilizing protocol that is specifically designed for the construction of an MLST. It improves other previous self-stabilizing solutions both for generality (arbitrary topology graphs $v s$. unit disk graphs or generalized unit disk graphs, respectively) and for approximation ratio, as it guarantees the number of its leaves is at least $1 / 3$ of the maximum one. The time complexity of our algorithm is $O\left(n^{2}\right)$ rounds.
\end{abstract}

\section{Introduction}

Multihop wireless ad hoc or sensor networks have neither fixed physical infrastructure nor central administration. They typically operate in a self-organizing manner that permits them to autonomously construct routing and communication primitives that are used by higher level applications. The construction of virtual backbones infrastructures usually makes use of graph related properties over the graph induced by communication capabilities (i.e. nodes represent machines, and edges represent the ability for two machines within wireless range to communicate) of the network. For example, a connected dominating set (CDS) is a good candidate for a virtual backbone since it guarantees reachability of every node yet preserves energy. The maximum leaf spanning tree (MLST) problem consists in constructing a spanning tree with the maximum number of leaves. Finding the MLST is tantamount to finding the minimum CDS: let $G=(V, E)$

\footnotetext{
* This work is supported in part by a Grant-in-Aid for Young Scientists ((B)22700074) of JSPS.

$\star \star$ This work is supported in part by Kayamori Foundation of Informational Science Advancement, a Grant-in-Aid for Scientific Research ((B)20300012) of JSPS, and "Global COE (Centers of Excellence) Program" of the Ministry of Education, Culture, Sports, Science and Technology, Japan.

${ }^{\star \star \star}$ This work is supported in part by ANR projects SHAMAN and ALADDIN
} 
be a graph and $c d s(G)$ be the size of the minimum CDS of $G$, then $|V|-c d s(G)$ is the number of leaves of the MLST of $G$ [1].

One of the most versatile techniques to ensure forward recovery of distributed systems and networks is that of self-stabilization [2]. A distributed algorithm is selfstabilizing if after faults and attacks hit the system and place it in some arbitrary global state, the system recovers from this catastrophic situation without external (e.g. human) intervention in finite time. As self-stabilization makes no hypothesis about the nature or the extent of the faults (self-stabilization only deals with the effect of the faults), it can also be used to deal with other transient changes while the network is being operated (topology change, message loss, spontaneous resets, etc.).

\subsection{Related Works}

In [3], Galbiati et al. proved that the MLST problem is MAX-SNP-hard, i.e., there exists $\varepsilon>0$ such that finding approximation algorithm ${ }^{5}$ with approximation ratio $1+\varepsilon$ is NP-hard. In [1], Solis-Oba proposed a 2-approximation algorithm, and in [4], Lu et al. proposed a 3 -approximation algorithm. Note that none of those algorithms $[1,4]$ is distributed, not to mention self-stabilizing.

Spanning tree construction is one of the main studied problems in self-stabilizing literature. One of the main recent trends in this topic is to provide self-stabilizing protocols for constrained variants of the spanning tree problem, e.g. [5], [6], [7], etc. None of those metrics give any guarantee on the number of leaves.

In [8], Guha et al. showed that the existence of an algorithm for finding the minimum CDS with approximation ratio $\alpha$ implies the existence of an algorithm of the MLST problem with approximation ratio $2 \alpha$. In turn, there exist self-stabilizing approximation algorithms for finding the minimum CDS. In [9], Kamei et al. proposed a self-stabilizing 7.6-approximation algorithm for the CDS problem in unit disk graphs, i.e., this algorithm is also an approximation algorithm for the MLST problem with approximation ratio 15.2 (by [8]). However, this algorithm [9] does not guarantee any approximation ratio in general topology networks. The subsequent work of Raei et al. [10] proposed a self-stabilizing $20\lfloor\ln R / \ln (2 \cos (\pi / 5))\rfloor$-approximation algorithm in generalized disk graphs where $R=r_{\max } / r_{\min }$ and $r_{\max }\left(\right.$ resp. $\left.r_{\min }\right)$ is the maximum (resp. minimum) transmission range. This algorithm is an approximation for the MLST problem with approximation ratio $40\lfloor\ln R / \ln (2 \cos (\pi / 5))\rfloor$. Again, this algorithm [10] does not guarantee any approximation ratio in general topology networks.

\subsection{Our contribution and outline of this paper}

We propose a fully distributed self-stabilizing approximation algorithm for the MLST problem on arbitrary topology networks. Its time complexity is $O\left(n^{2}\right)$ rounds. To our knowledge, our algorithm is the first self-stabilizing protocol that is specifically designed for the construction of MLST. It improves over previous self-stabilizing derivations both for generality (arbitrary topology graphs $v s$. unit disk graphs [9] (resp. gen-

\footnotetext{
${ }^{5}$ An approximation algorithm for the MLST problem is an algorithm that guarantees approximation ratio $\left|T_{\text {opt }}\right| /\left|T_{\text {alg }}\right|$, where $\left|T_{\text {alg }}\right|$ is the number of leaves obtained by the approximation algorithm in the worst case and $\left|T_{o p t}\right|$ is the number of leaves of the optimal solution.
} 
eralized disk graphs [10])) and for approximation ratio (3 vs. 15.2 [9] (resp. 40 $\lfloor\ln R /$ $\ln (2 \cos (\pi / 5))\rfloor[10]))$.

The improved approximation ratio permits to improve significantly the load and the energy consumed by the virtual backbone. The improved generality on the communication graph enables our scheme to be useful even in networks that cannot be modeled by (generalized) disk graphs (such as wired networks).

This paper is organized as follows. In Section 2, we formally describe the system model and the distributed MLST problem. In Section 3, we present our self-stabilizing approximation algorithm for the distributed MLST problem, and prove the correctness and analyze the time complexity of the proposed algorithm. Details of proofs are omitted because of limitation of space, and they will appear in the full paper. Concluding remarks can be found in Section 4.

\section{Preliminaries}

Let $V=\left\{P_{1}, P_{2}, \ldots, P_{n}\right\}$ be a set of $n$ processes and $E \subseteq V \times V$ be a set of bidirectional communication links in a distributed system. Each links is an unordered pair of distinct processes. Then, the topology of the distributed system is represented as an undirected graph $G=(V, E)$. We assume that $G$ is connected and simple. In this paper, we use "graphs" and "distributed systems" interchangeably. We assume that each process has unique identifier. By $P_{i}$, we denote the identifier of process $P_{i}$ for each process $P_{i}$.

We call subgraph of $G$ any graph $G^{\prime}=\left(V^{\prime}, E^{\prime}\right)$ such that $V^{\prime} \subseteq V, E^{\prime} \subseteq E$, and $\forall P_{i}, P_{j}$, $\left(P_{i}, P_{j}\right) \in E^{\prime} \Rightarrow P_{i}, P_{j} \in V^{\prime}$. By $N_{i}$, we denote the set of neighboring processes of $P_{i}$. For each process $P_{i}$, the set $N_{i}$ is assumed to be a constant. We define the degree of $P_{i}$ as the number of its neighbors. The degree of $P_{i}$ in the subgraph $G^{\prime}$ is the number of edges of $E^{\prime}$ incident to $P_{i}$. We assume that the maximum degree of $G$ is at least 3 . We define the distance between $P_{i}$ and $P_{j}$ as the number of the edges of the shortest path between them.

As communication model, we assume that each process can read the local state of neighboring processes. This model is called the state reading model. Although a process can read the local state of neighboring processes, it cannot update them; it can only update its local state.

A set of local variables defines the local state of a process. By $Q_{i}$, we denote the local state of each process $P_{i} \in V$. A tuple of the local state of each process $\left(Q_{1}, Q_{2}, \ldots, Q_{n}\right)$ forms a configuration of a distributed system. Let $\Gamma$ be a set of all configurations.

We say that $P_{i}$ is privileged in a configuration $\gamma$ if and only if at least one of the conditions of the algorithm is true and $P_{i}$ must change the value of its variables in $\gamma$. An atomic step of each process $P_{i}$ consists of following three sub-steps: (1) read the local states of all neighbors and evaluate the conditions of the algorithm, (2) compute the next local state, and (3) update the local state.

Executions of processes are scheduled by an external (virtual) scheduler called daemon. That is, the daemon decides which processes to execute in the next step. Here, we assume a distributed weakly fair daemon. Distributed means that, at each step, the daemon selects an arbitrary non-empty set of privileged processes, and selected processes executes the atomic step in parallel. Weakly fair means that every continuously privileged process will be eventually executed. 
For any configuration $\gamma$, let $\gamma^{\prime}$ be any configuration that follows $\gamma$. Then, we denote this transition relation by $\gamma \rightarrow \gamma^{\prime}$. For any configuration $\gamma_{0}$, a computation $E$ starting from $\gamma_{0}$ is a maximal (possibly infinite) sequence of configurations $E=\gamma_{0}, \gamma_{1}, \gamma_{2}, \ldots$ such that $\gamma_{t} \rightarrow \gamma_{t+1}$ for each $t \geq 0$.

Definition 1 (Self-Stabilization). Let $\Gamma$ be a set of all configurations. A system $S$ is self-stabilizing with respect to $\Lambda$ such that $\Lambda \subseteq \Gamma$ if and only if it satisfies the following two conditions:

- Convergence: Starting from an arbitrary configuration, a configuration eventually becomes one in $\Lambda$, and

- Closure: For any configuration $\lambda \in \Lambda$, any configuration $\gamma$ that follows $\lambda$ is also in $\Lambda$ as long as the system does not fail.

Each $\gamma \in \Lambda$ is called a legitimate configuration. Conversely, any configuration that is not legitimate is said illegitimate.

A spanning tree $T=\left(V^{\prime}, E^{\prime}\right)$ is any acyclic connected subgraph of $G$ such that $V^{\prime}=V$ and $E^{\prime} \subseteq E$. A leaf of a spanning tree is any process of degree one. Generally, the MLST problem is defined as follows.

Definition 2. The maximum leaf spanning tree is a spanning tree whose number of leaves is maximum.

We consider solving the MLST problem in distributed systems in this paper. We assume that each process does not know global information of the network. Under this assumption, we defined the distributed MLST problem as follows.

Definition 3. Let $G=(V, E)$ be a graph that represents a distributed system. Then, the distributed maximum leaf spanning tree problem is defined as follows.

- Each process $P_{i}$ must select a neighbor on $G$ or itself (if the father of $P_{i}$ is $P_{i}$, then $P_{i}$ is a root) as its father on a spanning tree $T_{m l}$ and output it, and

- The spanning tree $T_{m l}$ is a maximum leaf spanning tree of $G$.

\section{Proposed Algorithm}

Our algorithm SSMLST is based on the sequential approximation algorithm in [4].

We call tree any subgraph $T$ of $G$ that has no cycle and more than one process. We construct disjoint trees $T_{1}, T_{2}, \cdots$, where $T_{i}=\left(V_{i}, E_{i}\right), V=V_{1} \cup V_{2} \cup \cdots,\left|V_{i}\right|>1$, and $V_{i} \cap V_{j}=\emptyset$ for any $i$ and $j$. We call forest any set of trees $\left\{T_{1}, T_{2}, \cdots\right\}$. Note that some process $P_{i}$ can be alone and does not join the forest, i.e., $S_{i}=\left(\left\{P_{i}\right\}, \emptyset\right)$, in this case $P_{i}$ is called singleton.

Let $d_{k}(G)$ be the set of nodes that have degree $k$ on $G$, and let $\bar{d}_{k}(G)$ be the set of nodes that have degree at least $k$ on $G$.

Definition 4. ([4]) Let $T$ be a tree of $G$. If $\bar{d}_{3}(T)$ is not empty and every node in $d_{2}(T)$ is adjacent in $T$ to exactly two nodes in $\bar{d}_{3}(T)$, then let $T$ be leafy tree. Let $T_{1}, T_{2}, \cdots$ be disjoint trees on $G$. If each $T_{1}, T_{2}, \cdots$ is leafy, then $F=\left\{T_{1}, T_{2}, \cdots\right\}$ is a leafy forest. If $F$ is not a subgraph of any other leafy forest of $G$, then we call $F$ maximal leafy forest. 
In [4], Lu et al. showed the following theorem.

Theorem 1. ([4]) Let $F$ be a maximal leafy forest of $G$, and let $T_{m l}$ be a spanning tree of $G$ such that $F$ is a subgraph of $T_{m l}$. Let $T_{\text {span }}$ be any spanning tree of $G$. Then, $\left|d_{1}\left(T_{m l}\right)\right| \geq\left|d_{1}\left(T_{\text {span }}\right)\right| / 3$.

Our algorithm SSMLST first constructs a maximal leafy forest (MLF) of G, and then, it constructs a spanning tree $T_{m l}$ of $G$ that is a supergraph of the MLF. Hence, $T_{m l}$ is an approximation of the MLST with ratio 3 according to Theorem 1.

The proposed algorithm is a fair composition [11] of four layers:

1. In the first layer, each process $P_{i}$ computes its degree $D_{i}$ on $G$ and the maximum couple $M A X=\left(D_{0}, P_{0}\right)$ of degree $D_{0}$ and ID $P_{0}$ on $G$, where $\left(D_{i}, P_{i}\right)>\left(D_{j}, P_{j}\right) \equiv$ $\left[D_{i}>D_{j} \vee\left(D_{i}=D_{j} \wedge P_{i}>P_{j}\right)\right]$ for each process $P_{i}$ and $P_{j}$. For this layer, we can use a self-stabilizing leader election algorithm for arbitrary networks, for example [12] (The time complexity of [12] is $O(n)$ rounds.). In such an algorithm, the process with the minimum or the maximum ID is elected as a leader. It is modified to elect the process with the maximum value $\left(D_{0}, P_{0}\right)$ for our purpose.

2. The second layer SSMLF (see subsection 3.1) computes an MLF on $G$.

3. The third layer SSTN (see subsection 3.2) modifies the cost of each link based on the MLF.

4. The last layer computes a minimum cost spanning tree $T_{m l}$ based on the costs computed by SSTN. Such costs make $T_{m l}$ includes the MLF. For this layer, we can use one of existing self-stabilizing algorithms, e.g., [6] (The time complexity of [6] is $O\left(n^{2}\right)$ rounds. $)$.

\subsection{The Second Layer: Construction of the Maximal Leafy Forest}

We now propose a self-stabilizing algorithm called SSMLF that constructs of a maximal leafy forest (MLF) of $G$. The formal description of SSMLF is shown in Fig. 1.

Each process $P_{i}$ computes the following outputs:

- root $_{i}$ is set to $\emptyset$ if $P_{i}$ is a singleton. Otherwise, $P_{i}$ belongs to some tree $T$ and root $_{i}$ is set to the couple $\left(D_{r}, P_{r}\right)$, where $P_{r}$ is the root of the tree of $P_{i}$.

- father $r_{i}$ is set to the couple $\left(D_{j}, P_{j}\right)$ where $P_{j}$ is the father of $P_{i}$. If $P_{i}$ is neither a root nor a singleton, then $P_{j} \in N_{i}$. In this case, we say that " $P_{j}$ is a father of $P_{i}$ " and " $P_{i}$ is a child of $P_{j}$ ". In either cases ( $P_{i}$ is a singleton or the root of its tree), $P_{j}=P_{i}$. Note that, in SSMLF, each process $P_{i}$ distinguishes its incident link to father $_{i}$ as its parent-link in its tree.

- rank $k_{i}$ is the distance from $P_{i}$ to the root of its tree.

- MaxChildren $n_{i}$ : the expected number of children of $P_{i}$ in its tree. (We shall explain that later.)

In the following explanations, we call large tree any tree rooted at a process with a large couple of degree on $G$ and ID. Also, we call a child-candidate of process $P_{i}$ the neighbor of $P_{i}$ that may become a child of $P_{i}$ in the future, e.g., singleton, process belonging to a tree that is not larger than the tree of $P_{i}$, or process belonging to the tree 


\section{Constant (Input)}

$N_{i}$ : the set of neighbors of $P_{i}$ on $G$.

$D_{i}$ : the degree of $P_{i}$ on $G$ (an output from the first layer).

$M A X$ : the maximum couple of degree and ID on $G$ (an output from the first layer).

\section{Variable (Output)}

root $_{i}=\left(D_{r}, P_{r}\right)\left(\emptyset \leq\right.$ root $\left._{i} \leq M A X\right): P_{r}$ is the root of tree $T$ to which $P_{i}$ belongs.

father $_{i}$ : the couple $\left(D_{j}, P_{j}\right)$ of the father $P_{j}$ of $P_{i}$ on $T$.

$\operatorname{rank}_{i}$ : the distance from the root to $P_{i}$ on $T$.

MaxChildren $_{i}$ : the number of children and child-candidates on $T$.

\section{Macro}

MaxRoot $_{i}=\max \left\{\operatorname{root}_{j},\left(D_{i}, P_{i}\right) \mid P_{j} \in N_{i}\right\}$

MinRank $_{i}=\left\{\begin{array}{lr}-1 & \left(\text { In case that } \text { MaxRoot }_{i}=\left(D_{i}, P_{i}\right)\right) \\ \min \left\{\text { rank }_{j} \mid P_{j} \in N_{i} \wedge \text { root }_{j}=\text { MaxRoot }_{i}\right\}(\text { otherwise })\end{array}\right.$

CCand $_{i}=$

$$
\left\{P_{j} \in N_{i} \mid \text { root }_{j}=\emptyset \vee \text { root }_{j}<\text { MaxRoot }_{i} \vee\left(\text { root }_{j}=\text { MaxRoot }_{i} \wedge \operatorname{rank}_{j}>\text { MinRank }_{i}+2\right)\right\}
$$

CountMaxChildren $_{i}=\left\{\begin{array}{l}D_{i} \\ \mid\left\{P_{j} \in N_{i} \mid \text { father }_{j}=\left(D_{i}, P_{i}\right)|+| \text { In case that }\left(D_{i}, P_{i}\right)=\text { MAX) }\right.\end{array}\right.$

FCand $_{i}=\left\{P_{j} \in N_{i} \mid\right.$ rank $_{j}+1 \leq n \wedge\left(\right.$ MaxChildren $_{j} \geq 3 \vee$

$$
\left.\left.\left(\text { MaxChildren }_{j}=2 \wedge \text { father }_{j} \neq\left(D_{j}, P_{j}\right) \wedge \text { root }_{j}>\left(D_{j}, P_{j}\right)\right)\right)\right\}
$$

\section{Algorithm for process $P_{i}$ :}

do forever \{

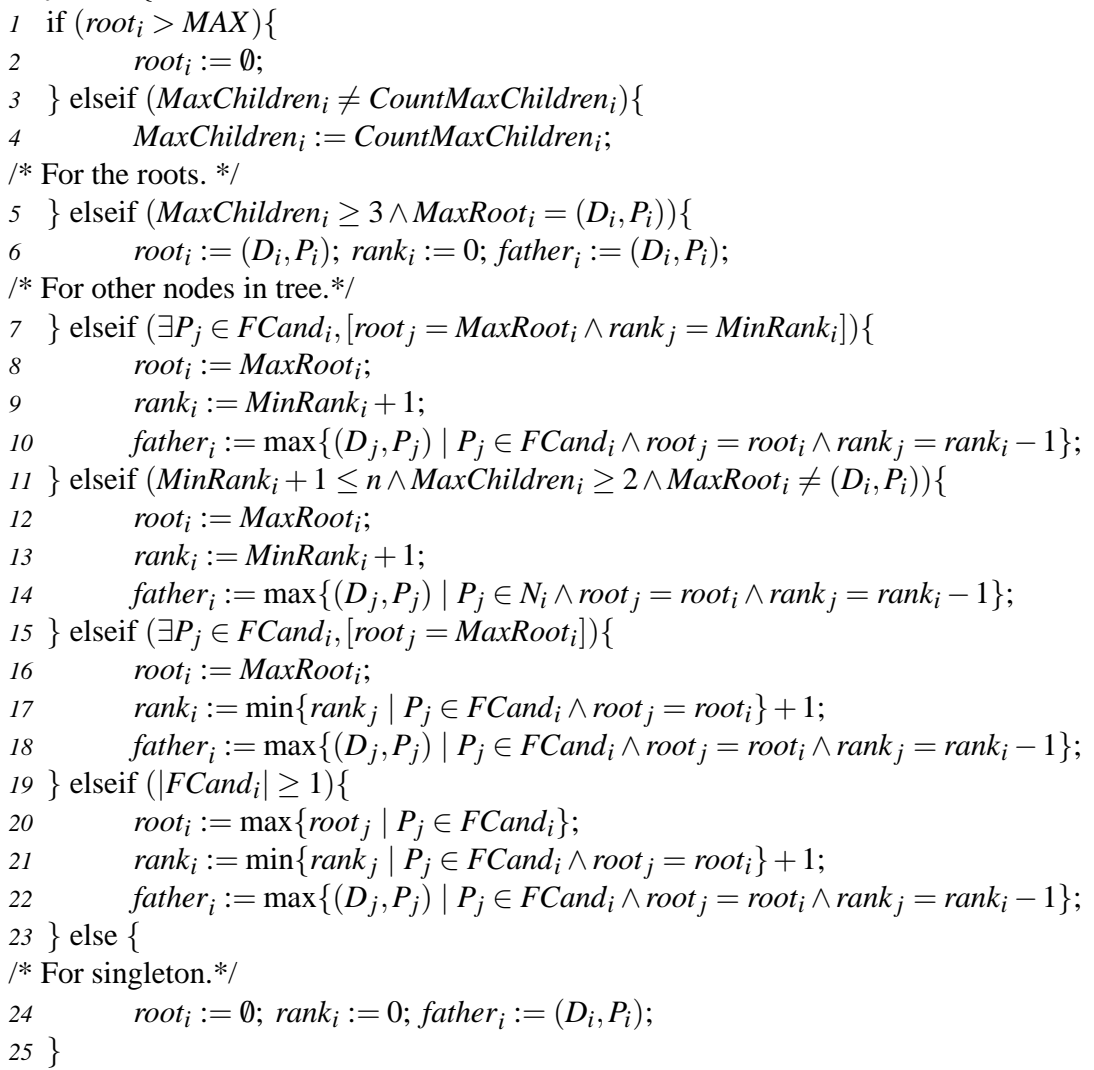

Fig. 1. SSMLF: Self-stabilizing algorithm for construction of the maximal leafy forest 
of $P_{i}$ that can minimize their height in the tree by changing its father to $P_{i}$. The expected number of children is the number of its children and child-candidates. Each process $P_{i}$ counts the number of expected number of children to make the tree leafy, and joins a tree as large as possible. That is, if $P_{i}$ is the root and its tree is larger than the one of its neighbors, then all its neighbors will join the tree of $P_{i}$.

According to Definition 4, SSMLF constructs of a maximal leafy forest (MLF) of $G$ by assigning its outputs following Definitions 5 and 6 .

Definition 5. Let sdeg $_{i}$ be the degree of process $P_{i}$ in its tree, i.e., sdeg $g_{i} \equiv \mid\left\{P_{j} \in\right.$ $N_{i} \mid$ father $\left._{j}=\left(D_{i}, P_{i}\right)\right\}|+|\left\{P_{j} \in N_{i} \mid\right.$ father $\left._{i}=\left(D_{j}, P_{j}\right)\right\} \mid$.

Definition 6. Let $T_{k}=\left(V_{k}, E_{k}\right)$ be a tree rooted at a process $P_{r}$ where $E_{k}$ is a set of links represented by the value of father $r_{i}$ of each process $P_{i}$ of $V_{k} \subseteq V$ and $s \operatorname{seg}_{r} \geq 3$ holds. Consider each process $P_{i}$ such that $s d e g_{i}=2$ on $T_{k}$. If sdeg $_{f} \geq 3$ and $s d e g_{j} \geq 3$ where father $_{i}=\left(D_{f}, P_{f}\right)$ and father ${ }_{j}=\left(D_{i}, P_{i}\right)$, then $T_{k}$ is leafy tree. If each tree $T_{1}, T_{2}, \cdots$ is disjoint and leafy on $G$, then the set $\left\{T_{1}, T_{2}, \cdots\right\}$ is a leafy forest $F$. If $F$ is not a subgraph of any other leafy forest, then $F$ is maximal leafy forest.

In order to evaluate its output variables, a process $P_{i}$ uses several macros:

- MaxRoot ${ }_{i}$ returns the largest value in the root-variables of $P_{i}$ and its neighbors.

- CCand $_{i}$ returns the set of child-candidates of $P_{i}$.

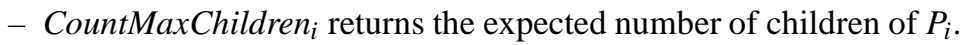

- FCand $i$ returns the father candidates of $P_{i}$, that is, the neighbors that $P_{i}$ can choose in order to make its tree leafy, i.e., process $P_{j}$ such that $r a n k_{j}$ is not obviously inconsistent and that has a chance of holding $s d e g_{j} \geq 3$.

- MinRank $k_{i}$ returns the rank-value of the (current or future) father of $P_{i}$ in its tree. (If $P_{i}$ is the root of its tree, then $\operatorname{MinRank}_{i}$ returns -1 so that $P_{i}$ sets $\operatorname{rank}_{i}$ to 0 .)

Now, we give more details about SSMLF. Consider a process $P_{i}$. In Lines 1-2, if the value of root $_{i}$ is obviously inconsistent, i.e., root $_{i}>M A X$, then $P_{i}$ resets its value to $\emptyset$. In Lines 3-4, $P_{i}$ updates MaxChildren ${ }_{i}$, if necessary. Then, if root r $_{i}$ and MaxChildren are correctly evaluated, $P_{i}$ must choose its status among root, internal node or leaf of a tree and singleton, and updates its variables root $_{i}$, rank $_{i}$, and father ${ }_{i}$ in consequence. That is what it does in Lines 5-24. Below, we detail these lines:

- In Lines 5-6, if MaxChildren $i \geq 3$ and $P_{i}$ can become a root of large tree, then $P_{i}$ becomes a root.

- In Lines 7-10, $P_{i}$ selects a process $P_{j}$ such that the distance from the root to $P_{j}$ is the minimum on a largest tree as father ${ }_{i}$ only if $P_{j}$ has a chance of holding $s d e g_{j} \geq 3$. That is, $P_{j} \in$ FCand $_{i}$.

- In Lines 11-14, if $P_{i}$ has a chance of holding $s d e g_{i} \geq 3$, then $P_{i}$ selects a process $P_{j}$ such that the distance from the root to $P_{j}$ is the minimum on a largest tree as father $_{i}$ even if $s \operatorname{seg}_{j}<3$, in order to make the tree leafy. By the condition of MinRank $k_{i}+$ $1 \leq n, P_{i}$ does not select a process $P_{j}$ such that the value of $r a n k_{j}$ is obviously inconsistent.

- In Lines 15-18, if $P_{i}$ does not have a chance of holding $s d e g_{i} \geq 3, P_{i}$ selects a process $P_{j}$ on a largest tree as father ${ }_{i}$ only if $P_{j}$ has a chance of holding $s d e g_{j} \geq 3$ by the condition $P_{j} \in$ FCand $_{i}$. 
- In Lines 19-22, if $P_{i}$ cannot belong to a largest tree, $P_{i}$ belongs to other tree.

- In Line 24, if $P_{i}$ cannot belong to any tree, $P_{i}$ becomes a singleton.

To show the correctness, we must first define the set of legitimate configurations of SSMLF. Such a definition is given in Definition 7 below:

Definition 7. A configuration of SSMLF is legitimate if and only if each process $P_{i}$ satisfies the following conditions:

- The connection by father $r_{i}$ represents the maximal leafy forest.

- If $P_{i}$ is on a tree $T$ of the maximal leafy forest, then the value of root ${ }_{i}$ of $P_{i}$ represents the root of $T$.

- If $P_{i}$ is not on any tree of the maximal leafy forest, then $P_{i}$ is called a singleton, father $_{i}=\left(D_{i}, P_{i}\right)$ and root ${ }_{i}=\emptyset$.

By $\Lambda_{f}$, we denote a set of legitimate configuration.

Theorem 2. The algorithm SSMLF is self-stabilizing with respect to $\Lambda_{f}$.

Proof Outline. The first part of the proof consists in proving that any terminal configuration of SSMLF is legitimate. So, in the following, we consider a configuration $\gamma$ where no process is privileged. In $\gamma^{\prime}$, each connected component $T$ represented by the value of father $_{i}$ is a tree or a singleton on $G$. Additionally, there exists no process such that the value of rank $k_{i}$ is obviously inconsistent in $\gamma^{\prime}$, i.e., for each process $P_{i}, r_{a n k} \leq$ $n-1$ holds. Then, each tree is rooted at a process $P_{i}$ such that father $_{i}=$ root $_{i}=\left(D_{i}, P_{i}\right)$ and sdeg $_{i}=$ MaxChildren $_{i}=D_{i}$, and $P_{0}$ such that $\left(D_{0}, P_{0}\right)=M A X$ is a root of a tree. Additionally, each singleton $P_{i}$ holds father $r_{i}=\left(D_{i}, P_{i}\right)$, root $_{i}=\emptyset$ and sdeg $_{i}=0$. Other processes $P_{i}$ selects a process $P_{j}$ such that $s d e g_{j} \geq 3$ as its father if $s d e g_{i}<3$, i.e., each tree $T$ represented by the values of fathers is leafy, and the leafy forest is maximal.

The second part of the proof consists in proving that eventually the configuration becomes $\gamma^{\prime}$ by the algorithm SSMLF.

For any configuration $\gamma$ and any computation starting from $\gamma$, each value of root $_{i}$ of each process $P_{i}$ on $G$ eventually become smaller than or equal to $M A X$, and $P_{0}$ such that $M A X=\left(D_{0}, P_{0}\right)$ decides the values of its each variable as a root of a tree and never change. After that, some processes $P_{i}$ eventually form the tree $T_{0}=\left(V_{0}, E_{0}\right)$ rooted at $P_{0}$ and never change the values of their each variable.

Let $G^{1}=\left(V^{1}, E^{1}\right)$ be an induced subgraph by $V \backslash V_{0}$. Let $\left(D_{\overline{1}}, P_{\overline{1}}\right)$ be the maximum couple among the processes on $G^{1}$ which are not neighboring to any process in $V_{0}$. If $D_{\overline{1}} \geq 3, P_{\overline{1}}$ eventually fixes the values of its variables to be a root forever. After that, some processes $P_{i}$ eventually form the tree $T_{1}=\left(V_{1}, E_{1}\right)$ rooted at $P_{\overline{1}}$ and stop changing the values of their variables.

By repeating this discussion, we have a series of trees $T_{0}=\left(V_{0}, E_{0}\right), T_{1}=\left(V_{1}, E_{1}\right)$, $\cdots, T_{k}=\left(V_{k}, E_{k}\right)$, where each process in $V_{0}, V_{1}, \cdots, V_{k}$ fixes the values of all its variables. Let $G^{k}$ be an induced subgraph by $V \backslash\left\{V_{0} \cup V_{1} \cup . . \cup V_{k}\right\}$. If $D_{\bar{k}}<3$ holds where the maximum couple on $G^{k}$ among the processes which are not neighboring to any process in $\left\{V_{0} \cup V_{1} \cup \cdots \cup V_{k}\right\}$, processes on $G^{k}$ cannot form any leafy tree, and they become singletons. Then, no process is privileged.

Theorem 3. The time complexity of algorithm SSMLF is $O\left(n^{2}\right)$ rounds. 


\subsection{The Third Layer: Modification of Edge Cost}

The third layer algorithm SSTN computes a network cost from the MLF computed by the second layer. In the fourth layer, the minimum spanning tree is computed based on this cost. The minimum spanning tree is the approximate solution of the MLST problem. Then, the edges in any tree must include the minimum spanning tree, and the intra-tree edges such that both endpoints are in the same tree must not be in the minimum spanning tree. Additionally, we would like to make the number of the connector edges between each tree as small as possible. The connector edges are selected from inter-tree edges such that its endpoints are not in the same tree.

Formal description of this layer SSMLF is shown in Fig. 2.

Definition 8. A configuration of SSTN is legitimate if and only if each edge satisfies the following three conditions.

- If e is a tree edge, then $W\left(P_{i}\right)\left[P_{j}\right]$ is 0 ,

- If e is an intra-tree edge, then $W\left(P_{i}\right)\left[P_{j}\right]$ is $\infty$, and

- If $e$ is an inter-tree edge, then $W\left(P_{i}\right)\left[P_{j}\right]$ is 1 .

By $\Lambda_{t}$, we denote a set of legitimate configuration.

\section{Constant (Input)}

$N_{i}$ : the set of neighbors on $G$.

root $_{i}$ : ID of the root of tree $T$ to which $P_{i}$ belongs on the MLF (output from Layer 2).

father $r_{i}$ ID of the father of $P_{i}$ in $T$ (output from Layer 2).

\section{Variable (Output)}

$W\left(P_{i}\right)\left[P_{j}\right]$ : new cost of the edge between $P_{i}$ and $P_{j} \in N_{i}$.

Algorithm for process $P_{i}$ :

do forever \{

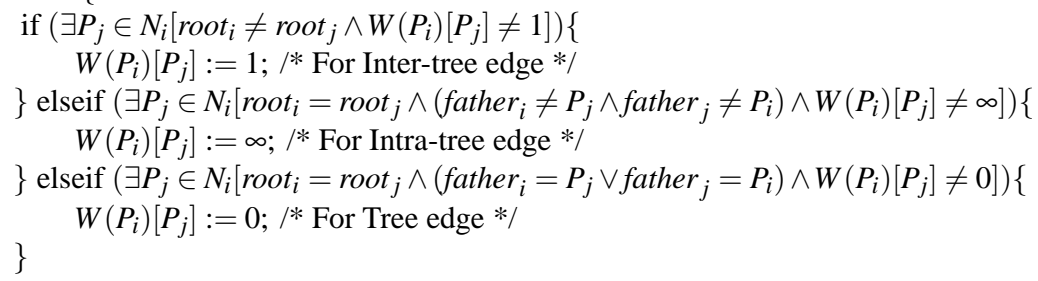

Fig. 2. SSTN: Self-stabilizing algorithm for transforming the network

Theorem 4. The algorithm SSTN is self-stabilizing with respect to $\Lambda_{t}$. The time complexity of algorithm SSTN is $O(n)$ rounds.

Each of the four layers stabilize in at most $O\left(n^{2}\right)$ rounds, hence we can conclude:

Theorem 5. The algorithm SSMLST is a self-stabilizing approximation algorithm for the MLST problem with approximation ratio 3. Its time complexity is $O\left(n^{2}\right)$ rounds. 


\section{Conclusion}

In this paper, we proposed a self-stabilizing distributed approximation algorithm for the MLST problem in arbitrary topology networks with approximation ratio 3. However, there exists a sequential solution [1] proposed by Solis-Oba that has approximation ratio 2 . Investigating the trade-off between approximation ratio and complexity of the self-stabilizing mechanism to achieve it is an immediate future work. Also, we would like to mention the importance to complement the self-stabilizing abilities of a distributed algorithm with some additional safety properties that are guaranteed when the permanent and intermittent failures that hit the system satisfy some conditions.

\section{References}

1. Solis-Oba, R.: 2-approximation algorithm for finding a spanning tree with maximum number of leaves. In: Proceedings of the 6th Annual European Symposium on Algorithms. LNCS 1461 (1998) 441-452

2. Tixeuil, S.: Self-stabilizing Algorithms. Chapman \& Hall/CRC Applied Algorithms and Data Structures. In: Algorithms and Theory of Computation Handbook, Second Edition. CRC Press, Taylor \& Francis Group (November 2009) 26.1-26.45

3. Galbiati, G., Maffioli, F., Morzenti, A.: A short note on the approximability of the maximum leaves spanning tree problem. Information Processing Letters 52(1) (1994) 45-49

4. Lu, H.I., Ravi, R.: Approximating maximum leaf spanning trees in almost linear time. Journal of algorithms 29 (1998) 132-141

5. Blin, L., Potop-Butucaru, M., Rovedakis, S.: Self-stabilizing minimum-degree spanning tree within one from the optimal degree. In: Proceedings of the 23th IEEE International Parallel and Distributed Processing Symposium. (2009)

6. Blin, L., Potop-Butucaru, M.G., Rovedakis, S., Tixeuil, S.: A new self-stabilizing minimum spanning tree construction with loop-free property. In: Proceedings of the 23rd International Symposium on Distributed Computing. LNCS 5805 (2009) 407-422

7. Butelle, F., Lavault, C., Bui, M.: A uniform self-stabilizing minimum diameter tree algorithm. In: Proceedings of the 9th International Workshop on Distributed Algorithms. LNCS 972 (1995) 257-272

8. Guha, S., Khuller, S.: Approximation Algorithms for Connected Dominating Sets. Algorithmica 20 (1998) 347-387

9. Kamei, S., Kakugawa, H.: A self-stabilizing distributed approximation algorithm for the minimum connected dominating set. In: Proceedings of the 9th IPDPS Workshop on Advances in Parallel and Distributed Computational Models. (2007) 224

10. Raei, H., Tabibzadeh, M., Ahmadipoor, B., Saei, S.: A self-stabilizing distributed algorithm for minimum connected dominating sets in wireless sensor networks with different transmission ranges. In: Proceedings of the 11th International Conference on Advanced Communication Technology. (2009) 526-530

11. Dolev, S.: Self-Stabilization. The MIT Press (2000)

12. Datta, A.K., Larmore, L.L., Vemula, P.: Self-stabilizing leader election in optimal space. In: Proceedings of the 10th International Symposium on Stabilization, Safety, and Security of Distributed Systems. LNCS 5340 (2008) 109 - 123 